\title{
CITATION INFLUENCE FOR JOURNAL AGGREGATES OF SCIENTIFIC PUBLICATIONS: THEORY, WITH APPLICATION TO THE LITERATURE OF PHYSICS $\dagger$
}

\author{
Gabriel Pinski $\ddagger$ and Francis Narin§ \\ Computer Horizons, Inc., 1050 Kings Highway North, Cherry Hill, NJ 08034, U.S.A.
}

\begin{abstract}
A self-consistent methodology is developed for determining citation based influence measures for scientific journals, subfields and fields. Starting with the cross citing matrix between journals or between aggregates of journals, an eigenvalue problem is formulated leading to a size independent influence weight for each journal or aggregate. Two other measures, the influence per publication and the total influence are then defined. Hierarchical influence diagrams and numerical data are presented to display journal interrelationships for journals within the subfields of physics. A wide range in influence is found between the most influential and least influential or peripheral journals.
\end{abstract}

\section{HISTORICAL BACKGROUND}

In the continuing attempt to assess, categorize and measure the rapidly increasing accumulation of knowledge, the ability to measure the influence of one area of activity on another would be a valuable asset. A quantitative scheme for evaluating the influence of scientific research in a given subfield of science or within a given institution could serve as a management aid in assessing the effectiveness of the scientific enterprise as well as providing data for science policy studies.

The use of citation analysis has been advocated and employed to provide a quantitative measure. The bibliographic use of citation data is not controversial; however, the merits and limitations of the bibliometric use of citation data has been the subject of active interest. The extent to which the data may be used and its application considered valid is a matter of controversy. Extreme positions extend from those who would employ a citation measure in the granting of tenure to an individual faculty member to those who deny the validity of any application of citation data beyond the use in literature search. Within this range of positions lies a spectrum of possible statistical applications from studies at the macro level dealing with large aggregates of publications to the micro level analysis of a single author or a single paper.

The appeal of citation analysis is attested to by its recurrence in the scientific literature during the last fifty years. In 1927, a modest paper published by Gross and Gross was the first to use citations to evaluate the importance of scientific journals; its concern was the adequacy of the chemical library collection at Pomona College [1]. Gross and Gross tabulated the references from the Journal of the American Chemical Society in 1926 and used these to rank the importance of the cited journals to American chemistry students. There followed at least twenty papers which used the same technique, the direct counting of citations from one or a small group of journals, as a measure of the importance of the cited journals to an area of science.

One of the earliest papers which uses journal to journal cross citations to measure the infiuence of one journal on another and one subfield on another is that of Cason and Lubotsky in 1936[2]. They stated that journal to journal citation analysis could be used "to secure a quantitative measure of the extent to which each psychological field influences and is influenced by each of the other psychological fields". More recently, Daniel and Louttit, in 1953, developed a cross citing matrix in psychology, measured the similarity of the individual journal citation patterns and performed what may well be the first formal clustering of scientific journals [3].

In 1964, M. M. Kessler, at MIT, formulated a journal cross citing matrix for physics journals and hypothesized that the probability that a journal carried a specific type of information could be deduced from his matrix [4].

Network theory concepts were used by Xhignesse and Osgood in 1967 to portray the relationships between journals and to measure their referencing similarities [5]. 
Due to the tediousness of the hand tabulation which was required for this kind of analysis, the full potential of citation studies was not realized during this period. The modern development of this field is largely due to the availability of citation and publication data in machine readable form; the Science Citation Index (SCI) published by the Institute for Scientific Information contains approximately 4 million citations from 400,000 publications in 2300 scientific journals in 1973. The results reported in the present paper are based on data extracted from the 1973 SCI.

In 1972 Garfield published an important work entitled "Citation Analysis as a Tool in Journal Evaluation" [6]. In it he gave explicit recognition to the policy implications inherent in such an analysis by using the subtitle "Journals can be Ranked by Frequency and Impact of Citations for Science Policy Studies". The citation based measure which Garfield calls the "impact factor" was introduced in this paper.

Also in 1972, Narin, Carpenter and Berlt used citations to map interrelationships among journals and among fields [7]. Some of the techniques introduced in that paper will be extended here. In a related paper, Carpenter and Narin applied a cluster analysis procedure to 288 journals in physics, chemistry and biomedicine, identifying many of the clusters with subdisciplinary subject areas[8].

\section{RATIONALE}

Previously used measures of influence all suffer from some defect which limits their use as evaluative measures.

The total number of publications of an individual, school or country is a measure of total activity only; no inferences concerning importance may be drawn.

The total number of citations to a set of publications, while incorporating a measure of peer group recognition, depends on the size of the set involved and has no meaning on absolute scale.

The journal "impact factor" introduced by Garfield is a size independent measure, since it is defined as the ratio of the number of citations the journal receives to the number of publications in a specified earlier time period. This measure, like the previous one, has no meaning on an absolute scale, but it in addition suffers from three more significant limitations. Although the size of the journal, as reflected in the number of publications, is corrected for, the average length of individual papers appearing in the journal is not. Thus, journals which publish longer papers, namely review journals, tend to have higher impact factors. In fact the nine highest impact factors obtained by Garfield were for review journals. This measure can therefore not be used to establish a "pecking order" for journal prestige.

The second limitation is that the citations are unweighted, all citations being counted with equal weight, regardless of the citing journal. It seems more reasonable to give higher weight to a citation from a prestigious journal than to a citation from a peripheral one. The idea of counting a reference from a more prestigious journal more heavily has also been suggested by KoCHEN [9].

The validity of any citation based measure necessarily assumes the free flow of citations in the referencing marketplace. A restraint such as a journal editorial policy restricting the number of times an author may refer to other journals would lead to the distortion of all citation based measures.

A third limitation is that there is no normalization for the different referencing characteristics of different segments of the literature: a citation received by a biochemistry journal, in a field noted for its large numbers of references and short citation times, may be quite different in value from a citation in astronomy, where the overall citation density is much lower and the citation time lag much longer.

In this paper three related influence measures are developed, each of which measures one aspect of a journal's influence, with explicit recognition of the size factor. These are: (1) the influence weight of the journal, a size independent measure of the weighted number of citations a journal receives from the other journals, normalized by the number of references it gives to other journals; (2) the influence per publication for the journal, which is the weighted number of citations each article, note or review in a journal receives from other journals; (3) the total influence of the journal which is the influence per publication times the total number of publications. 


\section{THE INFLUENCE WEIGHTING SCHEME}

\section{(a) Development of the weighting scheme}

1. The citation matrix.We are interested in describing the interactions among members of a set of publishing entities. These may, for example, be journals, institutions, individuals, fields of research, geographical subdivisions or levels of research methodology. The formalism to be developed is completely general in that it may be applied to any such set. To emphasize this generality we will refer to a member of a set as a unit rather than refer to a specific type of unit such as a journal.

The citation matrix is the fundamental entity which contains the information describing the flow of influence among units.

It has the form

$$
C=\left(\begin{array}{cccc}
C_{11} & C_{12} & \ldots & C_{1 n} \\
C_{21} & C_{22} & \ldots & C_{2 n} \\
\vdots & \vdots & \vdots \\
C_{n 1} & C_{n 2} & \ldots & C_{n n}
\end{array}\right)
$$

We will distinguish between the use of the terms "reference" and "citation" depending on whether we are discussing the issuing or receiving unit. Thus, a term $C_{i j}$ in the citation matrix indicates both the number of references unit $i$ gives to unit $j$ and the number of citations unit $j$ receives from unit $i$.

The time frame of a citation matrix must be clearly understood in order that a measure derived from it be given its proper interpretation. Suppose that the citation data are based on references issued in 1973. The citations received may be to papers in any year up through 1973. In general, the papers issuing the references will not be the same as those receiving the citations. Thus, any conclusions drawn from such a matrix assume an on-going, relatively constant nature for each of the units. For instance, if the units of study are journals, it is assumed that they have not changed in size relative to each other and represent a constant subject area. Journals in rapidly changing fields and new journals would therefore have to be treated with caution.

A citation matrix for a specific time lag may also be formulated. This would link publications in one time period with publications in some specified earlier time period.

2. Influence weights. For each unit in the set we want to extract from the citation matrix a measure of the influence of that unit. Because total influence is clearly a size dependent quantity, it is essential to distinguish between a size independent measure of influence, which we shall call the influence weight, and the size dependent total influence.

To make the idea of a size independent measure more precise, we may specify the following property of such a measure: If a journal were randomly subdivided into smaller entities, each would have the same measure as the parent journal.

The citation matrix may be though of as an "input-output" matrix with the medium of exchange being the citation. Each unit gives out references and receives citations; it is above average if it has a "positive citation balance", i.e receives more than it gives out. This reasoning provides a first order approximation to the weight of each unit, which is just

$$
W_{i}^{(1)}=\frac{\text { total number of citations to the } i \text { th unit from other units }}{\text { total number of references from the } i \text { th unit to other units }}
$$

This is the starting point for the iterative procedure for the calculation of the influence weights to be described below.

The denominator of this expression is the row sum

$$
S_{\mathrm{i}}=\sum_{j=1}^{n} C_{i j}
$$

corresponding to the th unit of the citation matrix; it may be thought of as the "target size" which this unit presents to the referencing world.

$W_{z}$ define the influence weight, $W_{i}$, of the $i$ th unit as 


$$
W_{i}=\sum_{k=1}^{n} \frac{W_{k} C_{k i}}{S_{i}}
$$

In the sum, the number of cites to the $i$ th unit from the $k$ th unit is weighted by the weight of $k$ th (referencing) unit. It is also divided by the target size $S_{i}$ of the unit $i$ being cited. The $n$ equations, one for each unit, provide a self consistent "bootstrap" set of relations in which each unit plays a role in determining the weight of every other unit. The following summarizes the derivation of those weights.

The equations defining the weights,

$$
W_{i}=\sum_{k=1}^{n} \frac{W_{k} C_{k i}}{S_{i}}, \quad i=1, \ldots, n
$$

are a special case of a more general system of equations which we may write in the form

$$
\sum_{k=1}^{n} W_{k} \gamma_{k i}-\lambda W_{i}=0, \quad i=1, \ldots, n
$$

Here $\gamma_{k i}=\left(C_{k i} / S_{i}\right)$ and we see that eqn (1) is a special case of eqn (2) corresponding to $\lambda=1$. As will be explained shortly the system of equations given in (1) will not, in general, possess a non-zero solution; only for certain values of $\lambda$ called the eigenvalues of the system, will there be non-zero solutions.

With our choice of target size, the value $\lambda=1$ is in fact an eigenvalue $\nmid$ so that eqn (1) itself does possess a solution.

Using the notation $\gamma^{T}$ for the transpose of $\gamma$, we have $\gamma_{i k}^{T}=\gamma_{k i}$; introducing the Kronecker delta symbol defined by

$$
\delta_{i k}= \begin{cases}1 & i=k \\ 0 & i \neq k\end{cases}
$$

we can then write

$$
\sum_{k=1}^{n}\left(\gamma_{i k}^{r}-\lambda \delta_{i k}\right) W_{k}=0
$$

This is a system of $n$ homogeneous equations for the weights. In order that a solution for such a system exists, the determinant of the coefficients must vanish. This gives an $n$th order equation for the eigenvalues

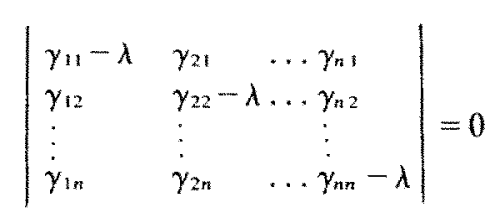

called the characteristic equation.

Only for values of $\lambda$ which satisfy this equation, does a non-zero solution for the $W$ 's exist. Moreover, eqn (3) does not determine the values of the $W_{k}$ themselves, but at best determines their ratios. Equivalently one may think of the eigenvalue equation as a vector equation for the vector unknown $\mathbf{W} \equiv\left\{W_{1}, \ldots, W_{n}\right\}$

$$
\gamma^{T} \cdot \mathbf{W}=\lambda \mathbf{W}
$$

from which it is clear that only the direction of $\mathbf{W}$ is determined.

The normalization or scale factor is then fixed by the condition that the size-weighted average of the weights is 1 , or 


$$
\frac{\sum_{1}^{n} S_{k} W_{k}}{\sum_{1}^{n} S_{k}}=1 .
$$

This normalization assures that the weight values have an absolute as well as a relative meaning, with the value 1 representing an average value.

Each root of the characteristic equation determines a solution vector or eigenvector of the equation, but the weight vector we are seeking is the eigenvector corresponding to the largest eigenvalue. This can be seen from the consideration of an alternative procedure for solving our system of equations, a procedure which also leads to the algorithm of choice.

Consider an iterative process starting with equal weights for all units. The values $W_{i}^{(0)}=1 \mathrm{can}$ be thought of as zeroth order approximations to the weights. The first order weights are then

$$
W_{i}^{(1)}=\frac{\sum_{k-1}^{n} C_{k i}}{S_{i}} .
$$

This ratio (total cites to a unit divided by the target size of the unit) is the simplest size-corrected citation measure and, in fact, corresponds to the impact measure used by Garfield. These values are then substituted into the right hand side of eqn (1) to obtain the next order of approximation. In general, the $m$ th order approximation is

$$
W_{i}^{(m)}=\sum_{k=1}^{n} \frac{W_{k}^{(m-1)} C_{k i}}{S_{i}}=\sum_{k=1}^{n} W_{k}^{(m-1)} \gamma_{k i}=\sum_{j=1}^{n}\left(\gamma^{m}\right)_{j i}
$$

The exact weights are therefore

$$
W_{i}=W_{i}^{(\infty)}=\sum_{j=1}^{n}\left(\lim _{m \rightarrow \infty} \gamma^{m}\right)_{j i}
$$

This provides the most convenient numerical procedure for finding the weights, the whole iteration procedure being reduced to successive squarings of the $\gamma$ matrix.

This procedure is closely related to the standard method for finding the dominant eigenvalue of a matrix. Since $\lambda=1$ is the largest eigenvalue, repeated squarings are all that is needed. If the largest eigenvalue had a value other than 1 , the normalization condition, eqn (6), would have to be reimposed with each squaring. Convergence to three decimal places usually occurs with six squarings, corresponding to raising $\gamma$ to the 64 th power.

It is instructive to examine the analytical results from some simple cases. The $2 \times 2 \gamma$ matrix has the form

$$
\gamma=\left(\begin{array}{ll}
\frac{C_{11}}{S_{1}} & \frac{C_{12}}{S_{2}} \\
\frac{C_{21}}{S_{1}} & \frac{C_{22}}{S_{2}}
\end{array}\right) .
$$

The target sizes are

$$
S_{1}=C_{11}+C_{12}, \quad S_{2}=C_{21}+C_{22}
$$

The eigenvalues of $\gamma$, and therefore also of $\gamma^{T}$, are $\lambda_{1}=1$ and

$$
\lambda_{2}=\frac{C_{11} C_{22}-C_{12} C_{21}}{\left(C_{11}+C_{12}\right)\left(C_{21}+C_{22}\right)}<1 . \dagger
$$

The case where $\lambda_{2}=1$ occurs only when there is no cross citation $\left(C_{12}=0=C_{21}\right)$, in which case the weights are undefined. 
The eigenvector of $\gamma^{T}$ corresponding to the eigenvalue 1 is $\left(\begin{array}{l}C_{21} \\ C_{12}\end{array}\right)$. Thus the ratios of the weights $W_{1}: W_{2}$ is $C_{21} / C_{12}$ or $W_{1}=N C_{21}, W_{2}=N C_{12}$.

The normalization factor $N$ follows from eqn (6).

$$
\begin{aligned}
\left(C_{11}+C_{12}\right) N C_{21}+\left(C_{21}+C_{22}\right) N C_{12} & =C_{11}+C_{12}+C_{21}+C_{22}=T \\
& =\text { Total No. of Citations }
\end{aligned}
$$

or

$$
N=\frac{T}{C_{11} C_{21}+2 C_{12} C_{21}+C_{12} C_{22}} .
$$

Alternatively, normalized weights can be found directly by finding $\gamma^{\infty}$. This is easily done by working with the diagonal form of $\gamma$,

$$
\gamma, \Lambda=\left(\begin{array}{cc}
1 & 0 \\
0 & \lambda_{2}
\end{array}\right)
$$

Since $\lambda_{2}<1$,

$$
\lambda_{2}<1, \Lambda^{\infty}=\left(\begin{array}{ll}
1 & 0 \\
0 & 0
\end{array}\right)
$$

The matrix which diagonalizes $\gamma$ by a similarity transformation is the matrix whose columns are the eigenvectors of $\gamma$. This matrix is

$$
S=\left(\begin{array}{cc}
C_{11}+C_{12} & C_{12} \\
C_{21}+C_{22} & -C_{21}
\end{array}\right)
$$

the first column being the eigenvector corresponding to $\lambda_{1}=1$, the second to $\lambda_{2}$.

The matrix $\gamma^{\infty}$ is then $\gamma^{\infty}=S \Lambda^{\infty} S^{-1}$. The weights are the column sums, $w_{i}=\gamma_{1 i}^{\infty}+\gamma_{2 i}^{\infty}$. We observe that the ratio of the weights, $C_{21} / C_{12}$, depends only on the cross citing between two units, not on self-citation; self-citation affects the absolute values of the weights only through the normalization condition. If the self-citation of one unit becomes large we can see that the weight of that unit approaches 1.

$$
\lim _{C_{11} \rightarrow \infty} W_{1}=\lim _{C_{11} \rightarrow \infty} \frac{C_{21}\left(C_{11}+C_{12}+C_{21}+C_{22}\right)}{C_{21} C_{11}+2 C_{21} C_{12}+C_{12} C_{22}}=1
$$

The $2 \times 2$ calculation can be used as an approximation for the weight of a single unit in the $n \times n$ problem. We form the condensed citation matrix

$$
\left(\begin{array}{ll}
C_{11} & C_{1 r} \\
C_{r 1} & C_{r r}
\end{array}\right)
$$

where

$$
C_{1 r}=\sum_{k=2}^{n} C_{1 k}, C_{r 1}=\sum_{k=2}^{n} C_{k 1}, C_{r r}=\sum_{k=2}^{n} \sum_{j=2}^{n} C_{j k}
$$

the "rest of the world", consisting of all units outside unit 1, is thus combined into a second "pseudo-unit". This provides only an approximation to the weight of unit 1 for the following reason: the details of individual interactions of unit 1 with each element of the pseudo-unit are lost, each element of the pseudo-unit carrying a common averaged weight. The way in which larger numbers of units interact may be glimpsed from the analytical result for the 3 unit case. 
In the 3 unit case the weight $W_{1}$ may be written

$$
W_{1}=R_{1}\left(\frac{\sum_{i} S_{i}}{\sum_{i} R_{i} S_{i}}\right)
$$

where

$$
S_{i}=C_{i 1}+C_{i 2}+C_{i 3}
$$

and

$$
R_{1}=C_{21} C_{31}+C_{32} C_{21}+C_{23} C_{31}
$$

$R_{2}$ and $R_{3}$ follow by cyclic permutation of the three indices. The expression in parentheses is the normalization factor, common to each of the weights. The ratios of the three weights are given by $W_{1}: W_{2}: W_{3}=R_{1}: R_{2}: R_{3}$. It is worth examining the structure of $R_{1}$. The first term, $C_{21} C_{31}$ is the product of the direct citations to unit 1 . The other two terms each contain "indirect citations", $C_{32} C_{21}$ being the product of the number of cites 1 receives from 2 weighted by the number that 2 receives from 3 . Note that $R_{1}$ contains no references from unit 1 .

The weights derived by the above process serve as a natural basis for construction of an influence hierarchy. In such a hierarchy the unit with highest weight, the most "weighty", appears at the base, with lighter weight units appearing above it. This is opposite from the ordering in an organizational hierarchy diagram.

There is no ambiguity in the ordering based on the weights as there is in a hierarchy based on pairwise comparisons. In the latter case, one starts with two units. The one receiving more cites from the other goes below it. The next unit is then compared with each of the first two and its position determined. However, there is no guarantee of transitivity among 3 or more units and so an ordering arrived at in this way is not unambiguous. With numerical weights we can go beyond an ordinal ranking to a calibrated scale, giving meaning to the distance between the elements of the hierarchy.

\section{(b) Influence measures and scaling}

The influcnce weights which are generated by the weighting scheme are a measure of influence per reference. It is natural to expect that of two publications appearing in journals which have equal influence weights, (or appearing in the same journal), the longer one will have the greater influence. The influence per publication is defined as the weighted number of cites (each cite weighted by the weight of the referencing journal) a publication receives. From eqn (1), the total number of wcighted cites for the ith journal is

$$
\sum_{k=1}^{n} W_{k} C_{k i}=W_{i} S_{i}
$$

To get the influence per publication divide by the yearly number of publications, $P_{i}$. Multiplication of $W_{i}$ by $S_{i} / P_{i}$ therefore yields the desired measure. Restating this result, the product

$$
\text { Influence Weight } \times \frac{\text { References }}{\text { Publication }}=\text { Influence } / \text { Publication }
$$

represents the influence per publication for the journal.

The third of our influence measures is the total influence defined by

Influence $/$ Pub. $\times$ No. of Pubs $=$ Total Influence. 
Two journals could have the same influence weight and contain publications of the same average length and yet have widely different total influence, solely due to the difference in number of publications.

These points may be illustrated by examining the results for four physics journals shown in Table 1.

Table 1. Influence measures for four physics journals

\begin{tabular}{lcccrr}
\hline & Inf Wt & Ref/Pub & Inf/Pub & Pubs & Tot Infl \\
\hline Phys Rev & 1.42 & 18.6 & 26.4 & 3648 & 96321 \\
Ann Physics & 1.66 & 17.4 & 29.0 & 147 & 4256 \\
Phys Rev L & 3.42 & 11.1 & 38.1 & 897 & 34186 \\
Rev M Phys & 2.10 & 116.9 & 245.8 & 18 & 4424 \\
\hline
\end{tabular}

Physical Review Letters has the highest influence weight. It has the smallest number of references per publication, being a letter journal, but still has higher influence per publication than Physical Review and Annals of Physics. Reviews of Modern Physics, containing only lengthy review articles has a reference per publication value of from six to ten times that of the other journals and so has by far the largest influence per publication. It should be obvious that the dominant factor here is the length of the average publication. A review journal usually has fewer than 30 publications per year so that its total influence is not large. We may contrast the total influence of Reviews of Modern Physics with that of Physical Review and Physical Review Letters.

It is worth comparing the set of figures tabulated for Physical Review with those for Annals of Physics. Annals of Physics has a somewhat larger influence weight and influence per publication than does Physical Review, but has less than $5 \%$ of the total influence of the Physical Review. It is clear that this effect is due to the number of publications appearing in Physical Review as opposed to Annals of Physics.

We see that the three measures-influence weight, influence per publication and total influence-reflect different information about a journal.

\section{(c) The aggregation problem}

The influence weight of any unit depends on the set with which it is interacting. A weight $W=1$ represents an average weight. The size weighted average of the weights of all the units must be 1 . If there are only two units and the weight of one is above 1 , the weight of the second must be below 1 .

The manner in which journals are grouped together with other journals has an unavoidable effect on journal weighting. The weight of a journal is completely dependent upon the choice of the set of journals with which it is interacting. The Journal of Chemical Physics (JCP) will obviously have a different weight when evaluated as a chemistry journal as opposed to as a physics journal.

There is however a more subtle type of aggregation problem which affects the weighting scheme. We recognize that chemical physics is a true linking area between physics and chemistry, with the direction of net information flow being from physics to chemistry. We would therefore expect that the weight of the chemical physics grouping would lie between that of chemistry and physics. When the weighting procedure is applied to aggregated chemistry, physics and chemical physics journals it turns out that the weight of the chemical physics grouping is actually higher than that of both physics and chemistry. This is explained by the fact that chemical physics is a small grouping dominated by the large, influential JCP while the physics and chemistry sets are composed of a large number of diverse quality journals. Thus, when a weighting calculation is performed involving individual journals from different fields, there are two intermingled effects at work. There is the overall influence flow from one field to another in which most single journals play, by themselves, only a small role. Superimposed upon this is the flow of influence among journals within a field. Within a field, journals may be thought of as "competing" for the same publications; it is then tempting to associate influence with quality. 
The situation is different when one is dealing with journals belonging to different fields. Different fields represent different areas of knowledge which we have no intention of ordering in importance. It must therefore be emphasized that differences in influence weights involving journals belonging to different fields or subfields, or involving the fields or subfields themselves should not be associated with quality.

\section{APPLICATION TO PHYSICS JOURNALS}

The influence measures for physics journals are listed in Table 2 . The calculations were performed as part of a larger study involving all fields of science. Journals in astronomy and astrophysics and in geophysics were classified in the field of earth and space science rather than in physics and, therefore, are not included in this paper. Multidisciplinary journals such as Science, Nature and the Proceedings of the Royal Society (London) also do not appear.

Table 2. Influence measures for physics journals

\begin{tabular}{|c|c|c|c|c|c|}
\hline Physics & $\begin{array}{l}\text { Infl } \\
\text { Wt }\end{array}$ & $\begin{array}{c}\text { Refs/ } \\
\text { Pub }\end{array}$ & $\begin{array}{l}\text { Infl/ } \\
\text { Pub }\end{array}$ & Pubs & $\begin{array}{l}\text { Tot } \\
\text { Infl }\end{array}$ \\
\hline \multicolumn{6}{|c|}{ General physics } \\
\hline Act Phys Au & 0.24 & 12.4 & 3.0 & 54 & 164 \\
\hline Act Phys Ch & 0.47 & 10.5 & 4.9 & 23 & 113 \\
\hline Act Phys $\mathrm{H}$ & 0.33 & 13.4 & 4.4 & 42 & 184 \\
\hline Adv Physics & 1.12 & 125.1 & 140.4 & 11 & 1545 \\
\hline Am J Phys & 0.94 & 3.0 & 2.9 & 323 & 924 \\
\hline Ann Physics & 1.66 & 17.4 & 29.0 & 147 & 4256 \\
\hline Ann Physik & 1.95 & 8.8 & 17.2 & 49 & 842 \\
\hline Ann $R$ Nucl & 0.45 & 116.8 & 52.7 & 12 & 632 \\
\hline Can J Phys & 0.86 & 13.3 & 11.5 & 339 & 3898 \\
\hline Cont Phys & 0.29 & 20.1 & 5.8 & 20 & 117 \\
\hline Czec J Phys & 0.22 & 9.3 & 2.0 & 193 & 392 \\
\hline Fortschr Ph & 0.37 & 32.1 & 11.7 & 16 & 187 \\
\hline Helv Phys A & 1.15 & 4.4 & 5.1 & 180 & 918 \\
\hline I J Physics & 0.34 & 7.6 & 2.6 & 74 & 189 \\
\hline Ivuz Fiz & 0.01 & 5.0 & 0.0 & 435 & 13 \\
\hline J Phys & 0.59 & 13.1 & 7.7 & 1672 & 12908 \\
\hline JETP Letter & 1.25 & 9.8 & 12.2 & 349 & 4268 \\
\hline Lett Nuov C & 0.32 & 8.1 & 2.6 & 609 & 1583 \\
\hline Nuov Cim & 1.04 & 13.8 & 14.3 & 449 & 6425 \\
\hline P Pm S Jap & 0.74 & 10.4 & 7.6 & 820 & 6257 \\
\hline Philos Mag & 1.97 & 12.7 & 24.9 & 228 & 5673 \\
\hline Phys Lett & 1.60 & 7.5 & 12.1 & 1622 & 19578 \\
\hline Phys Norveg & 0.73 & 12.8 & 9.4 & 12 & 112 \\
\hline Phys Rev & 1.42 & 18.6 & 26.4 & 3648 & 96307 \\
\hline Phys Rev L & 3.42 & 11.1 & 38.1 & 897 & 34185 \\
\hline Phys Scr & 0.17 & 15.8 & 2.8 & 149 & 411 \\
\hline Phys Today & 0.41 & 17.2 & 7.0 & 33 & 232 \\
\hline Physica & 0.85 & 13.0 & 11.1 & 309 & 3433 \\
\hline Prog T Phys & 0.55 & 17.0 & 9.4 & 396 & 3711 \\
\hline Rep Pr Phys & 0.27 & 117.6 & 31.6 & 29 & 917 \\
\hline Rev M Phys & 2.10 & 116.9 & 245.8 & 18 & 4424 \\
\hline Rev Ro Phys & 0.08 & 8.2 & 0.7 & 113 & 75 \\
\hline Sov J Nuc R & 0.52 & 16.2 & 8.4 & 315 & 2637 \\
\hline Sov Ph JE R & 2.35 & 10.6 & 24.9 & 598 & 14902 \\
\hline Z Phys & 1.11 & 14.5 & 16.0 & 346 & 5529 \\
\hline \multicolumn{6}{|c|}{ Nuclear and particle physics } \\
\hline Nucl Phys & 0.93 & 21.8 & 20.2 & 1209 & 24446 \\
\hline Usp Fiz Nau & 0.20 & 63.9 & 12.6 & 64 & 806 \\
\hline \multicolumn{6}{|c|}{ Solid state physics } \\
\hline J Phys Ch S & 1.24 & 16.2 & 20.1 & 252 & 5078 \\
\hline Phys St Sol & 0.31 & 11.9 & 3.7 & 1496 & 5505 \\
\hline Sol St Comm & 0.51 & 9.3 & 4.7 & 777 & 3675 \\
\hline Sov Ph Se R & 0.14 & 13.9 & 2.0 & 479 & 944 \\
\hline Sov Ph SS R & 0.58 & 8.2 & 4.8 & 905 & 4308 \\
\hline \multicolumn{6}{|c|}{ Chemical physics } \\
\hline Chem P Lett & 0.39 & 11.1 & 4.3 & 969 & 4241 \\
\hline J Chem Phys & 1.36 & 18.2 & 24.8 & 1448 & 35931 \\
\hline J Magn Res & 0.11 & 14.5 & 1.5 & 190 & 291 \\
\hline
\end{tabular}


Table 2(contd)

\begin{tabular}{|c|c|c|c|c|c|}
\hline Physics & $\begin{array}{l}\text { Infl } \\
\text { Wt }\end{array}$ & $\begin{array}{c}\text { Refs/ } \\
\text { Pub }\end{array}$ & $\begin{array}{l}\text { Infl/ } \\
\text { Pub }\end{array}$ & Pubs & $\begin{array}{l}\text { Tot } \\
\text { Inft }\end{array}$ \\
\hline Molec Phys & 0.35 & 17.8 & 6.2 & 289 & 1780 \\
\hline Surf Sci & 0.37 & 17.1 & 6.4 & 324 & 2077 \\
\hline \multicolumn{6}{|c|}{ Applied physics } \\
\hline Appl Phys I. & 1.89 & 7.2 & 136 & 498 & 6748 \\
\hline Cryogenics & 0.40 & 7.8 & 3.1 & 151 & 465 \\
\hline Energy Conv & 0.45 & 6.8 & 3.1 & 16 & 49 \\
\hline Ferroelectr & 0.20 & 29.1 & 5.7 & 23 & 131 \\
\hline High Temp R & 0.07 & 7.6 & 0.5 & 263 & 137 \\
\hline High Temp S & 0.25 & 15.8 & 3.9 & 42 & 163 \\
\hline I J PA Phys & 0.06 & 8.5 & 0.5 & 348 & 181 \\
\hline IEFE J Q $F \mid$ & 0.70 & 15.6 & 10.9 & 159 & 1730 \\
\hline Infrar Phys & 0.50 & 6.7 & 3.3 & 35 & 117 \\
\hline I Appl Phys & 1.23 & 11.3 & 13.9 & $\mid 05\rceil$ & 14619 \\
\hline J.L.Temp Ph & 0.22 & 18.2 & 4.0 & 174 & 696 \\
\hline J Mecanique & 0.56 & 5.9 & 3.3 & 22 & 72 \\
\hline I Mech Phys & 2.95 & 7.6 & 22.5 & 22 & 496 \\
\hline J Vac Sci T & 0.42 & 13.6 & 5.7 & 156 & 883 \\
\hline Jap J A Phys & 0.34 & 7.4 & 2.5 & 433 & 1074 \\
\hline Metrologia & 0.80 & 8.9 & 7.2 & 24 & 172 \\
\hline Nuct Instr & 0.65 & 8.8 & 5.7 & 627 & 3593 \\
\hline Phil Res $\mathrm{R}$ & 0.86 & 17.3 & 14.8 & 37 & 548 \\
\hline Phil Tech R & 0.49 & 8.0 & 3.9 & 36 & 140 \\
\hline Prib Tokhn & 0.23 & 2.8 & 0.6 & 537 & 349 \\
\hline Rep NRL Pro & 0.10 & 1.6 & 0.2 & 241 & 39 \\
\hline Rev In Haut & 0.12 & 13.0 & 1.6 & 28 & 46 \\
\hline Rev Phys Ap & 0.19 & 9.8 & 1.8 & 60 & 110 \\
\hline Rev Sci Ins & 1.72 & 5.5 & 9.5 & 434 & 4127 \\
\hline Sov Ph Tp R & 0.77 & 6.4 & 4.9 & 367 & 1809 \\
\hline Thin Sol Fi & 0.15 & 11.8 & 1.8 & 248 & 436 \\
\hline Vacuum & 0.22 & 8.7 & 2.0 & 77 & 152 \\
\hline Vakuum-Tech & 0.02 & 9.9 & 0.2 & 28 & 7 \\
\hline Vide & 0.19 & 4.1 & 0.8 & 43 & 34 \\
\hline \multicolumn{6}{|c|}{ Fuids and Plasmas } \\
\hline Ann R Fluid & 0.26 & 34.1 & 8.8 & 16 & 140 \\
\hline I Fluid Mec & 1.31 & 10,0 & 13.1 & 236 & 3092 \\
\hline J Plasma Ph & 0.39 & 10.5 & 4.1 & 71 & 290 \\
\hline Nucl Fusion & 0.56 & 12.2 & 6.8 & 79 & 536 \\
\hline Phys Fluids & 1.39 & 9.9 & 13.9 & 362 & 5014 \\
\hline Plasma Phys & 0.56 & 8.1 & 4.5 & 124 & 556 \\
\hline \multicolumn{6}{|l|}{ Acoustics } \\
\hline Acustica & 0.37 & 6.8 & 2.2 & 110 & 245 \\
\hline IEEE Audio & 0.22 & 6.6 & 1.4 & 76 & 109 \\
\hline IFFE SOM UI & 0.55 & 120 & 6.6 & 51 & 338 \\
\hline 1 Acoust 50 & 1.50 & 10,0 & 15.0 & 350 & 5250 \\
\hline$J$ Aud Eng $S$ & 0.17 & 5.6 & 0.9 & 71 & 66 \\
\hline I Sound Vib & 0.27 & 7.4 & 2.0 & 191 & 382 \\
\hline Sov Ph AC R & 0.43 & 7.7 & 3.3 & 108 & 361 \\
\hline Ultrasonics & 0.29 & 6.4 & 1.8 & 32 & 58 \\
\hline \multicolumn{6}{|l|}{ Optics } \\
\hline Anpl Optics & 0.82 & 9.5 & 7.8 & 430 & 3337 \\
\hline Jont Sox & 1.95 & 12.1 & 23,5 & 232 & 5464 \\
\hline 1 Phot Sei & 0.13 & 13.0 & 1.7 & 43 & 74 \\
\hline Optic Acta & 0.42 & 10.7 & 4.5 & 63 & 282 \\
\hline Optik & 0.60 & 7.3 & 4.3 & 107 & 463 \\
\hline Phot Sci Fn & 0.13 & 13.3 & 1.7 & 91 & 1.59 \\
\hline Photogr Eng & 0.49 & 2.1 & 1.0 & 85 & 88 \\
\hline 7h $\mathrm{Np} \mathrm{Fotog}$ & 0.06 & 6.5 & 0,4 & 78 & 28 \\
\hline \multicolumn{6}{|c|}{ Miscellaneous phystes } \\
\hline Ann 1 Hen $A$ & 0.99 & 7.6 & 7.6 & 40 & 303 \\
\hline Comm Math $\mathrm{P}$ & 1.41 & 7.5 & 10.5 & 122 & 1286 \\
\hline 5 Comput $\mathrm{Ph}$ & 0.19 & 8.1 & 1.5 & 128 & 192 \\
\hline J Math Phys & 1.54 & 9.2 & 14.1 & 290 & 4092 \\
\hline Phys Cond $\mathrm{M}$ & 0.60 & 24.2 & 14.5 & 31 & 450 \\
\hline
\end{tabular}


Journal relationships within the set of physics journals are shown graphically in a set of influence maps. Each influence map is a representation of journal influence within a subfield or related group of subfields. The following conventions apply to these maps: (1) A solid rectangle is used to represent journals within the subfield or subfields being presented on a given map. SCI journals abbreviations are used for all journals. The area of a rectangle is proportional to the size of a journal, as measured by the number of articles, notes and reviews in the Corporate Index of the $S C I$ in 1973. (2) The vertical scale shows influence per publication for each journal on a log scale. Weights for a set of units tend to be distributed in a log uniform rather than in a uniform manner and so use of a log scale results in less crowding for the lower weight units. Only journals reporting original research appear on the maps. Review journals, because of the large size of their individual publications, tend to have exceptionally high influence per publication. Their role in the literature is different from that of journals that report primarily original research; it is not, therefore, appropriate to compare the influence per publication of review and research journals. (3) The horizontal direction is used to separate either different subfields appearing on the same map, or journals with different specific foci. Journals in the same column tend to be more similar to each other than to journals in neighboring columns. (4) Arrows are directed from a journal to the other journals, exclusive of itself, to which it refers most frequently. Usually, two arrows are drawn from each journal showing the two other journals that are most frequently referenced; occasionally three are given if the number of references to the second and third are close, or there may be only one if a single arrow best characterizes the referencing priority of the journal. An arrow with a full head is used for a first arrow (largest number of references) while a half head is used for a second or third arrow. A dotted arrow is used for a secondary arrow which is considerably weaker than the primary arrow. If an arrow is directed to a journal which is not classified as being in the field under study, the "target" journal may be treated in one of several ways: (a) If the journal is of exceptional importance to the journals within the field of interest it will appear in a dashed rectangle located on the vertical scale by its influence per publication. An
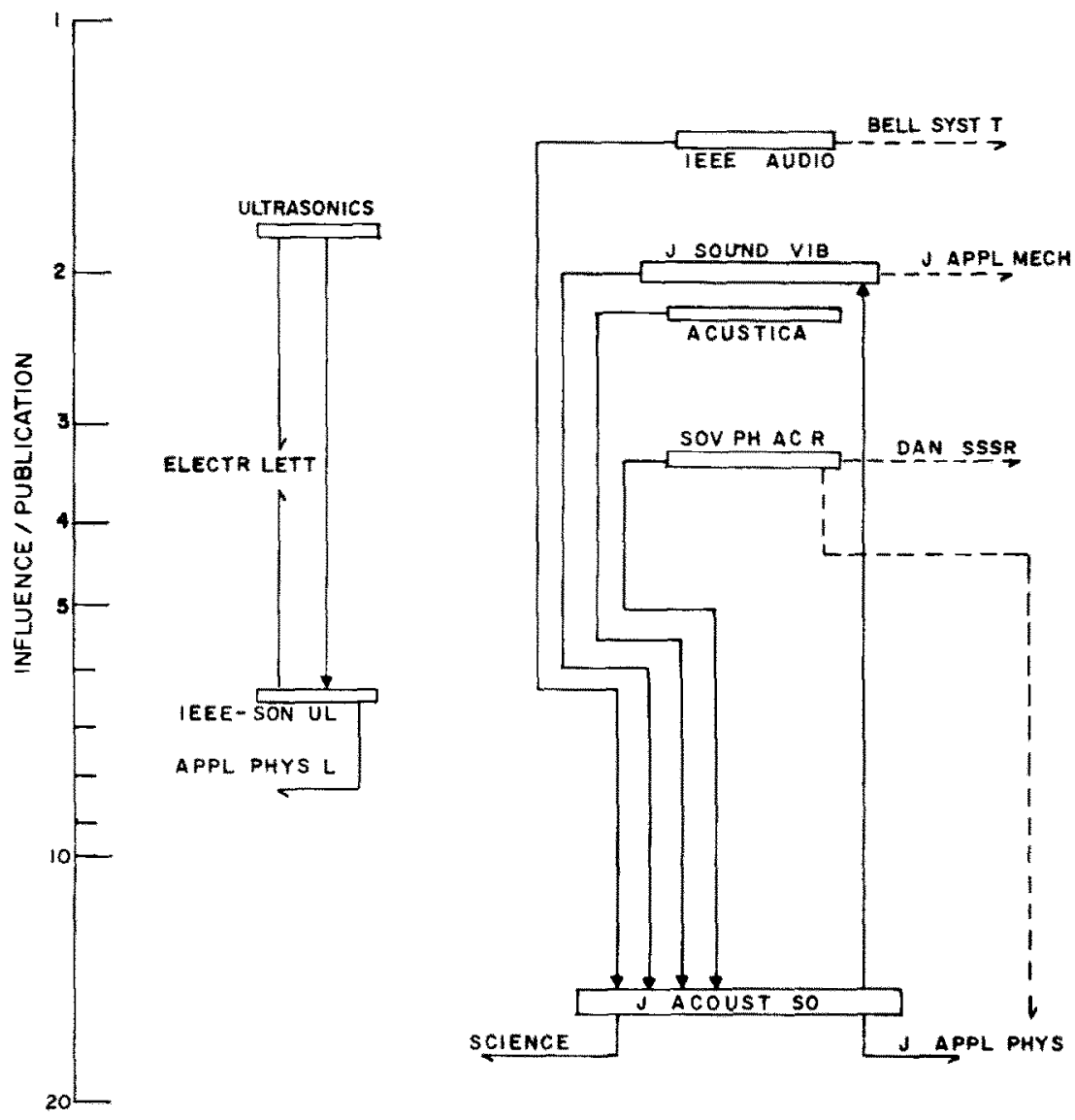

Fig. 1. Influence map for acoustics journals. 
example of this is the appearance of Physical Review Letters on the map of fluids and plasmas journals. (b) Arrows directed out of the subfield to journals which are not of central importance to the field are generally short arrows leading to the unenclosed journal name. For this case there is no significance to the vertical placement of the cited journal.

The fields of acoustics (Fig. 1) and optics (Fig. 2) are each dominated by their respective American Institute of Physics publications, the Journals of the Acoustical Society of America and the Journal of the Optical Society of America. The Optical Society journal has an influence per publication which is three times that of the nearest optical journal. In Fig. 2 the photographic science journals appear to the right of the central column of optics journals while the journal Infrared Physics is at the left. In the acoustics map, the journals dealing with ultrasound are separated from the main acoustics column. It is a common phenomenon that the most influential journal in a subfield refers frequently to large, more general journals. This is seen in the references from the Acoustical Society journal to the Journal of Applied Physics and Science, and from the Optical Society journal to the Physical Review.

The map for journals in fluids and plasmas is shown in Fig. 3. Here there are two journals, Physics of Fluids and the Journal of Fluid Mechanics which have almost equal values for the influence per publication. The journals in plasma physics and the journal Nuclear Fusion are
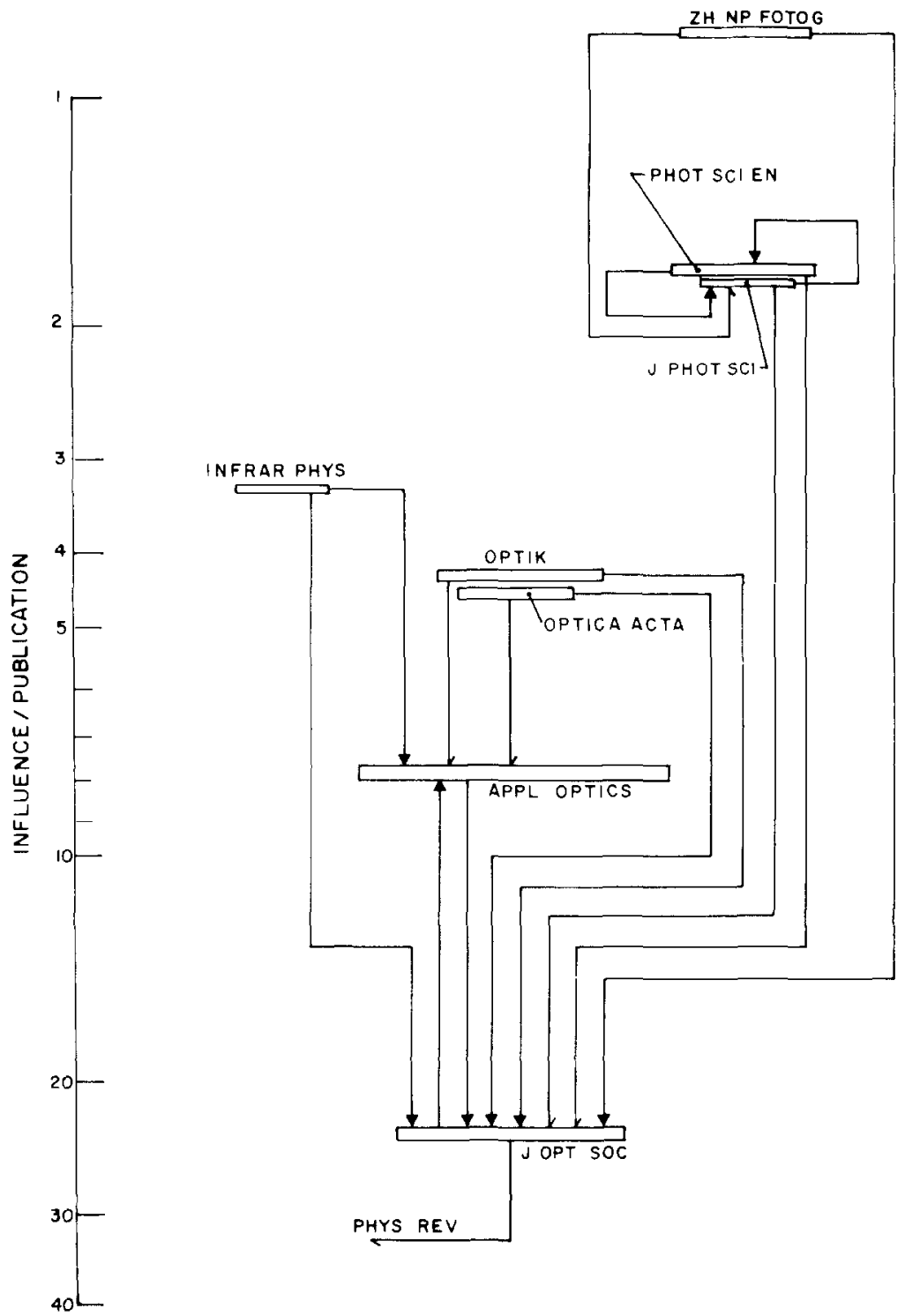

Fig. 2. Influence map for optics journals. 


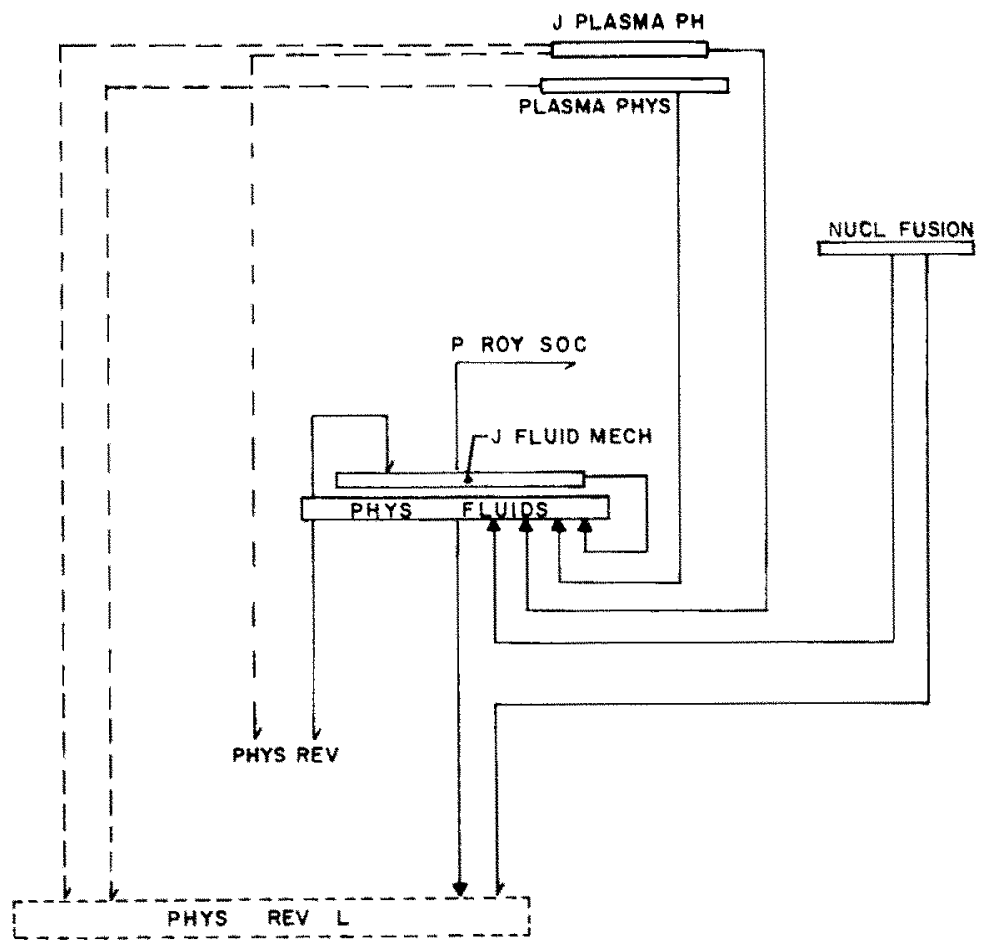

Fig. 3. Influence map for fluids and plasmas journals.

separated towards the right. It is apparent that much work of interest to this subfield is published in the general physics literature. Physics of Fluids refers most often to Physical Review Letters and next to the Physical Review, while most of the others in the group give their second arrow to one of the general journals.

The subfields of physics vary in the extent to which their literature is self-contained. While acoustics and optics each have a literature which is highly self-contained, solid state and nuclear physics research are dispersed throughout the general physics literature in addition to appearing in journals specific for these subfields. The citation analysis of these subfields of physics is impeded by the journal section problem. Since 1970 the Physical Review has been divided into four sections. Section C covers nuclear structure, D covers particles and fields, B covers solid state while A includes the remainder of physics research. During 1964 and 1965 there were only two sections, with B covering nuclear and elementrary particle physics and A solid state and other topics. In all other years there was no sectional division. If we use citation data for all previous years then it is clear that citations to the different subfields cannot be segregated. The sections of the Physical Review were therefore recombined giving a single massive general journal. Similar problems exist for the journals Nuovo Cimento, Physics Letters, Journal of Physics and for Nuclear Physics which is now split between nuclear structure in one section and particles and fields in the other. The result is that the largest, most central physics journals are forced into the general physics category. Only two journals were classified as nuclear and particle physics journals.

Figure 4 contains the general physics journals together with solid state, nuclear and mathematical physics. The general journals are in the central column with the letter journals displaced slightly towards the left. The Physical Review is referred to most frequently by a large majority of journals on this map. Arrows are most closely related to total influence so that this fact is explained in large part by the size of the journal. Since, as has been noted in Section 3(b), Annals of Physics has a higher influence per publication, it lies below Physical Review in the hierarchy. The same is true for Physical Review Letters which has the highest influence per publication of all (non-review) physics journals. 


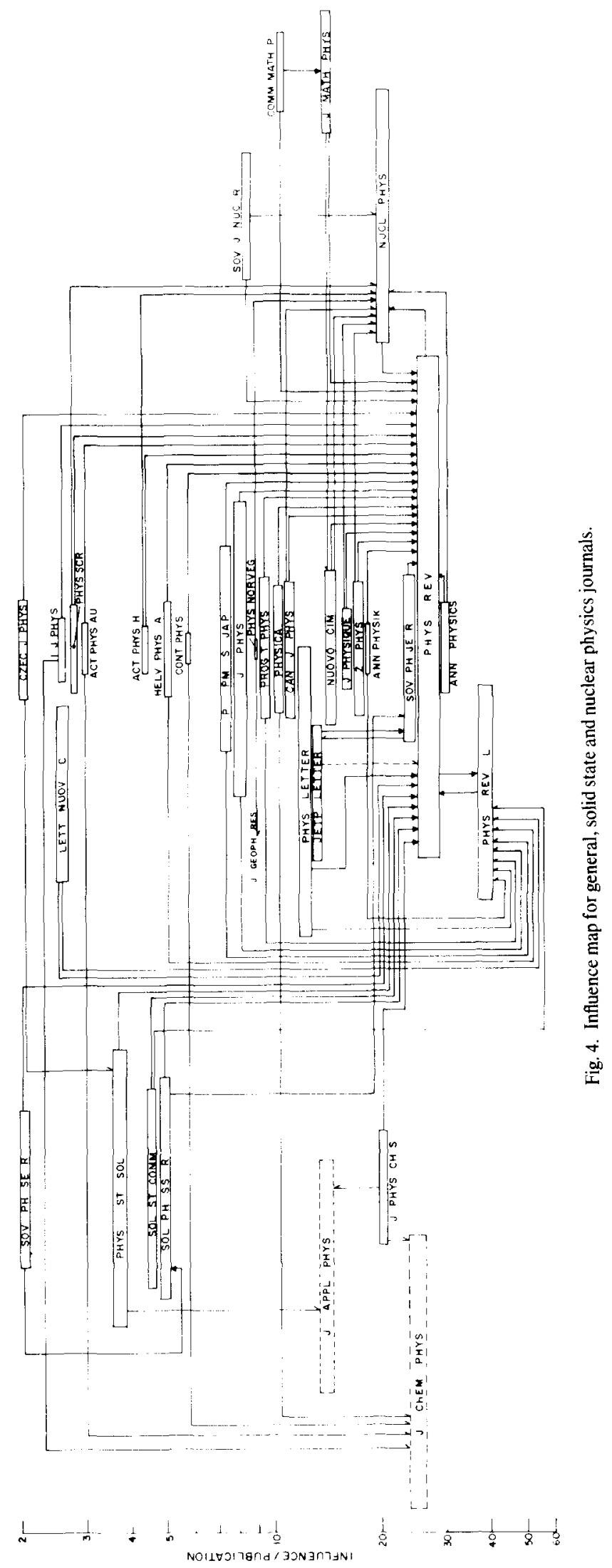




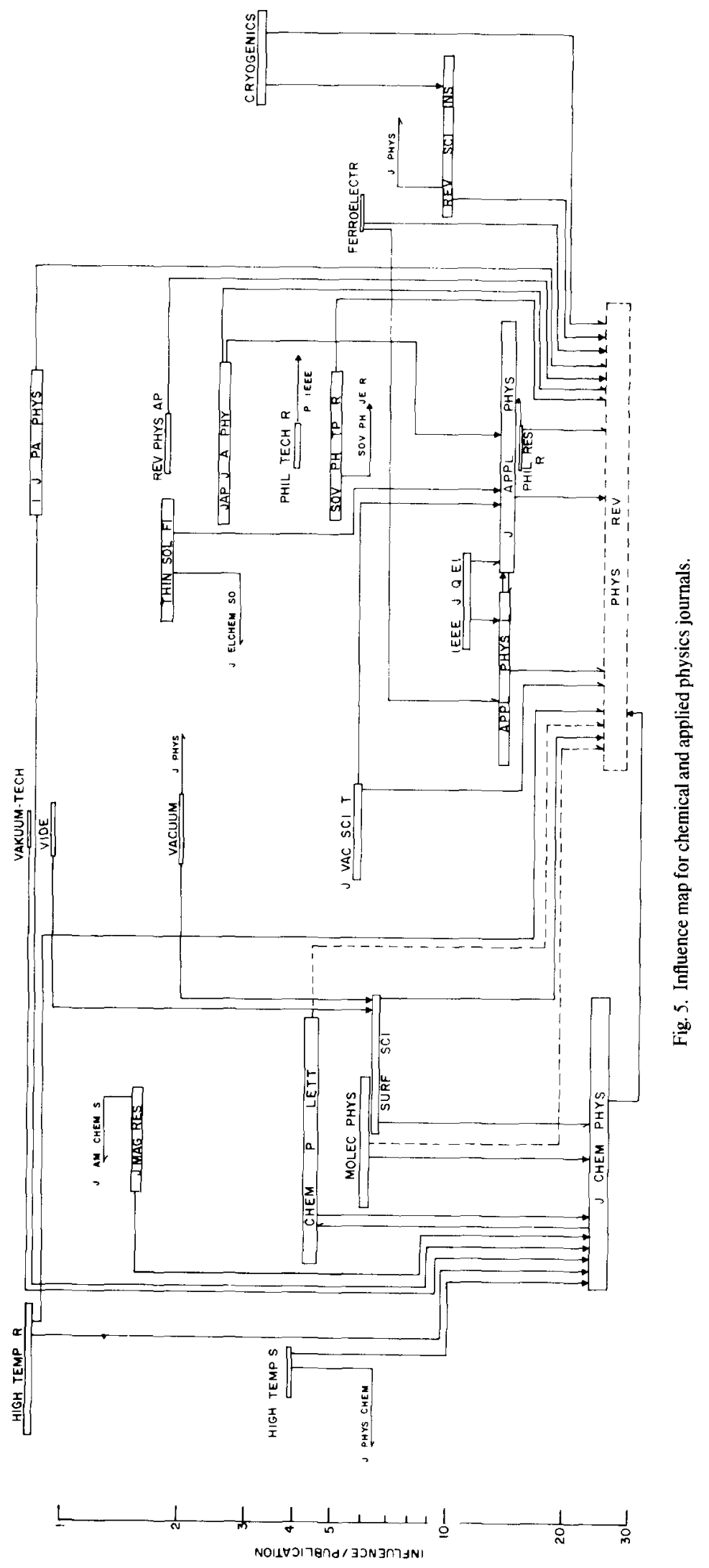


Applied physics and chemical physics journals appear in Fig. 5. While the Iournal of Applied Physics and Applied Physics Letters are leading journals in the applied area, most applied journals refer to them less frequently than they do to the Physical Review. The Journal of Chemical Physics is cited highly by a wide range of journals including general physics journals and those in chemical, solid state and applied physics as well as general and physical chemistry journals.

\section{CONCLUSIONS}

An influence weighting methodology has been developed and applied to 103 journals in physics. The introduction of three distinct measures allows for the separation of size effects arising from varying numbers of papers in a journal as well as those from varying lengths of papers. A wide range in influence is apparent between the influence of an average publication in a highly influential journal as opposed to an average publication in a peripheral journal.

This set of measures provides someone outside a field with objection criteria to assess the influence of an aggregate of scientific publications. A funding agency with its need to allocate scarce resources, or one concerned with science policy decisions should find this methodology to be a valuable evaluative tool.

\section{REFERENCES}

[1] P. L. K. Gross and E. M. Gross, College libraries and chemical education. Science 1927, 66, 385.

[2] H. CASON and M. Lubotsky, The influence and dependence of psychological journals on each other. Psych. B. 1936, 33, 95.

[3] R. S. Daniel and C. M. Loutrit, Professional Problems in Psychology. Prentice Hall. New York (1953).

[4] M. M. KESSLER, Some Statistical Properties of Citations in the Literature of Physics (Edited by M. E. STEVENS), Statistical Association Methods in Mechanized Documentation (Symposium Proceedings, 1964), Washington Nal. Bur. Std. 193-198 (1965).

[5] L. V. XHIGNESSE and C. E. OSGOOD, Bibliographic citation characteristics of the psychological journal network in 1950 and 1960. Am. Psychol. 1967, 22, 778 .

[6] E. GarfiELD, Citation analysis as a tool in journal evaluation. Science 1972, 178, 471.

[7] F. NARIN, M. P. CarPEnTER and N. C. BerLt, Interrelationships of scientific journals, JASIS 1972, 23, 323.

[8] M. P. Carpenter and F. NARIN, Clustering of scientific journals. JASIS 1973, 24, 425.

[9] M. Kochen, Principles of Information Retrieval, p. 83. Wiley, New York (1974). 